\title{
Identifying Loose Connective and Muscle Tissues on Histology Images
}

\author{
Claudia Mazo $^{1}$, Maria Trujillo ${ }^{1}$, and Liliana Salazar ${ }^{2}$ \\ 1 School of Computer and Systems Engineering \\ 2 Department of Morphology \\ Universidad del Valle
}

\begin{abstract}
Histology images are used to identify biological structures present in living organisms - cells, tissues, and organs - correctly. The structure of tissues varies according to the type and purpose of the tissue. Automatic identification of tissues is an open problem in image processing. In this paper, the identification of loose connective and muscle tissues based on morphological tissue information is presented.

Image identification is commonly evaluated in isolation. This is done either by eye or via some other quality measure. Expert criteria — by eye - are used to evaluate the identification results. Experimental results show that the proposed approach yields results close to the real results, according to expert opinion.
\end{abstract}

\section{Introduction}

The development of digital technologies has made available, to physicians and biologists, digital cameras connected to microscopes for image capture in order to preserve observed samples. Thus, large repositories of images are gathered of histological samples and allow automatic identification of tissues.

Connective and muscle are two of the four basic body tissues. The connective tissue is divided into: loose and dense. The dense connective is classified into: regular and irregular. The muscle tissue is divided into: striated or skeletal, smooth, striatal heart [6]. In this paper, we focus on identify loose connective and muscle tissues.

The identification of loose connective and muscle tissues is an open problem because of the close relation between them and the difficulty to demarcate the boundaries of each one. This process starts with a segmentation of each of the tissues and their refinement to eliminate additional information.

Automatic segmentation of the loose connective and muscle tissues involves several problems: the hard boundary between loose connective and muscle tissues in areas where they interrelate, the presence of red blood cells in some samples, the similarity between smooth muscle and dense regular connective tissue, among others.

In this paper, an automatic segmentation of loose connective and muscle tissue approach based on morphological information is presented. Obtained results of the largest eigenvalue of structure tensor along with the red and the green

J. Ruiz-Shulcloper and G. Sanniti di Baja (Eds.): CIARP 2013, Part II, LNCS 8259, pp. 174-180 2013.

(C) Springer-Verlag Berlin Heidelberg 2013 
color channels are combined in the K-means clustering for identifying loose connective tissue and obtaining a first approximation of the identification of muscle tissue. Finally morphological operations, thresholding, subtraction of images and thresholds are used to select areas of muscle tissue and refine results of muscle identification. Experimental results show that the proposed approach identifies correctly loose connective and muscle tissue in histological images. The rest of the paper is organised as follows. The proposed segmentation approach is presented in Section II. Experimental validation is included in Section III. Finally, conclusions are in Section IV.

\section{Proposed Approach}

The loose connective tissue is characterised by abundant fluid and tissue basic substance, dispersed structure and more numerous cells than the fibers. The smooth muscle is composed of spindle-shaped cells with a central nuclei which lack transverse striations while exhibiting weak longitudinal grooves. The heart muscle central nuclei and ramifications and interconnections between the fibers. The overall muscle tissue is more dense and compact. The example images of the tissues are illustrated in Fig. 1.

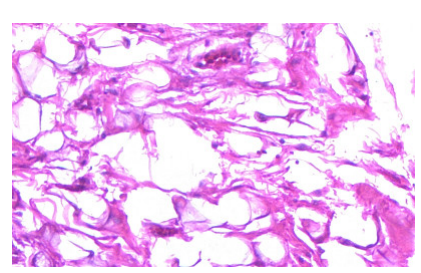

Connective Tissue

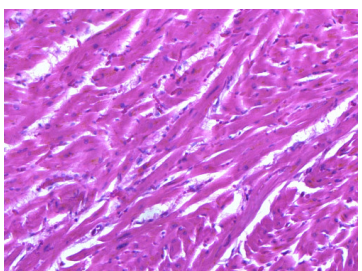

Heart Muscle Tissue

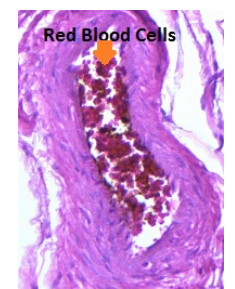

Smooth Muscle Tissue

Fig. 1. Example of loose connective and muscle tissue

Obtained borders with the largest eigenvalue of structure tensor algorithm along with the red and the green color channels are used as input into the Kmeans algorithm in order to obtain loose connective tissues and muscle tissues segmentation. Morphological operations, thresholding and region thresholds are used to refine areas of muscle tissue obtained with the K-means.

\subsection{Identification of Loose Connective Tissue}

Initially, the Structure tensor using the maximum eigenvalues [1, 2] is calculated. This algorithm allows us to obtain the nuclei of the cells [7. Finally, the identification of loose connective is performed using the K-means algorithm. 
Largest Eigenvalue of Structure Tensor. The largest eigenvalue of structure tensor [5] is described as follows. Given the red color channel of an image, the red channel is selected since it has the greatest information contains cell nuclei, the structure tensor $J_{0}$ is defined as the outer product of the gradient vector $\nabla I$ :

$$
J_{0}=\nabla I \nabla I^{T}=\left(\begin{array}{cc}
I_{x}^{2} & I_{x} I_{y} \\
I_{x} I_{y} & I_{y}^{2}
\end{array}\right),
$$

where $I^{T}$ symbolised the transpose of I. $J_{0}$ is extended to the linear structure tensor by a convolution of the components of $J_{0}$ with a Gaussian kernel $K_{p}$ (Gaussian smoothing)in order to consider neighbouring information:

$$
J_{\rho}=J_{0} * K_{\rho}=\left(\begin{array}{ll}
j_{11} & j_{12} \\
j_{12} & j_{22}
\end{array}\right) .
$$

The matrix $J_{p}$ has orthonormal eigenvectors $v_{1}$ and $v_{2}$ with $v_{1}$ parallel to

$$
\left(\frac{2 j_{11}}{j_{11}+j_{22}-\sqrt{j_{11}-j_{22}^{2}+4 j_{12}^{2}}}\right) .
$$

The eigenvalues are given by

$$
\mu_{1}=\frac{1}{2}\left[j_{11}+j_{22}+\sqrt{j_{11}-j_{22}^{2}+4 j_{12}^{2}}\right],
$$

and

$$
\mu_{2}=\frac{1}{2}\left[j_{11}+j_{22}-\sqrt{j_{11}-j_{22}^{2}+4 j_{12}^{2}}\right] .
$$

The eigenvalues describe the average contrast in the eigen-directions within a neighbourhood of size $(\rho)$. The vector $v_{1}$ indicates the orientation with the highest red value fluctuations, while $v_{2}$ gives the preferred local orientation, the coherence direction. Furthermore, $\mu_{1}$ and $\mu_{2}$ serve as descriptors of local structure. Isotropic areas are characterised by $\mu_{1} \cong \mu_{2}$, straight edges gives $\mu_{1}$ $\gg \mu_{1}=0$, corner by $\mu_{1} \geq \mu_{2} \gg 0$ [5].

The structure tensor of an image is a method of analysing the edge structure in an image. Eigenvectors point in the direction orthogonal and across the local edge, with the Eigenvalues indicating the strength of the directional intensity change. The larger eigenvalue shows the strength of the local image edges, the corresponding eigenvector points across the edge (in gradient direction). In this way we get a grayscale image as a result.

The K-means Algorithm. To perform the segmentation of images identifying: loose connective tissue, muscle tissue and light regions, the K-means algorithm is used [3]. A psudocode of the K-means algorithm is sketched as follows: 


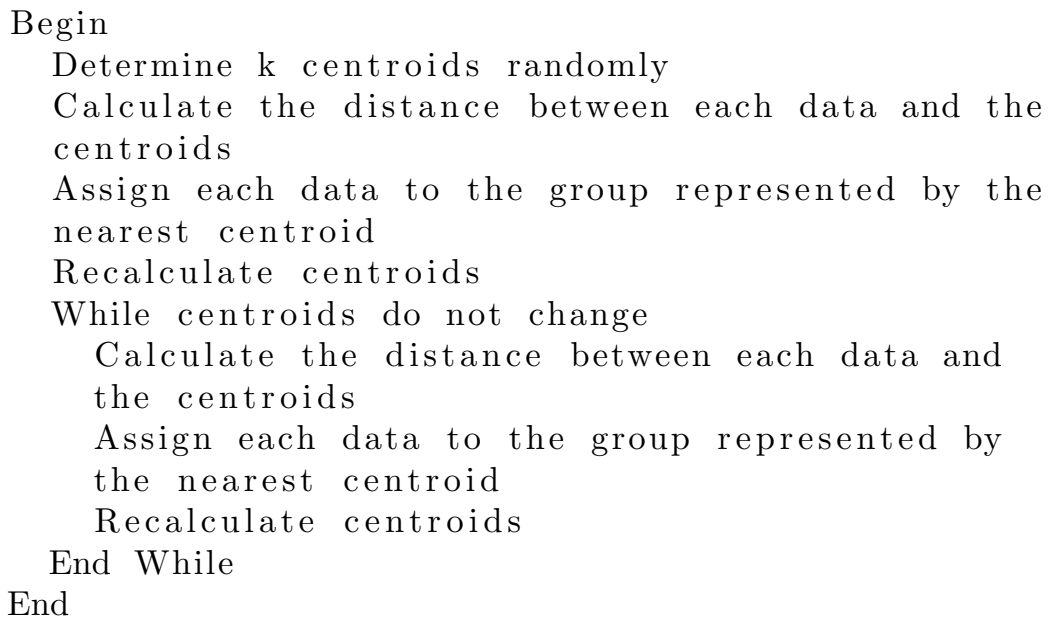

\subsection{Identification of Muscle Tissue}

A first segmentation of muscle was performed in the previous step for the Kmeans. However, it is necessary to refine the results since it included the loose connective tissue in some areas and the red blood cells. To improve the segmentation result will apply three steps: first, erosion [8] is performed on the muscle segmented image. Second, the result of the erosion is subjected to a thresholding, regions under threshold1 are removed. The size of the regions is controlled by the Flood-fill algorithm 4. Finally the red blood cells are removed with a process of thresholding and morphological operation.

Erosion. A problem of the segmented images is the presence of irrelevant details - from the point of view of size. To eliminate these small islands and bumps the segmented image is erode [8]. The erosion and morphological operation is defined as:

$$
\begin{gathered}
I \ominus C=\left\{x \in E \mid C_{x} \subseteq I\right\}, \\
C_{x}=\{c+x \mid c \in C\}, \forall_{x} \in E,
\end{gathered}
$$

let $E$ be a Euclidean space $\mathbb{R}^{d}$ or an integer grid $\mathbb{Z}^{d} . I$ is a binary image in E. $C_{z}$ is the translation of $B$ by the vector $z$. For erosion to be satisfied that the set of all points $x$, such that $C$ forward $x$, are contained in $I$.

Threshold. After performing the erosion an image without irrelevant size areas is obteined, however there are irrelevant areas that are not eliminated since have a larger size, but are reduced. To eliminate these areas a thresholding process is performed, then regions under a threshold are removed. The size of the regions is 
controlled by the Flood-fill algorithm 4]. A psudocode of the Flood-fill algorithm is sketched as follows:

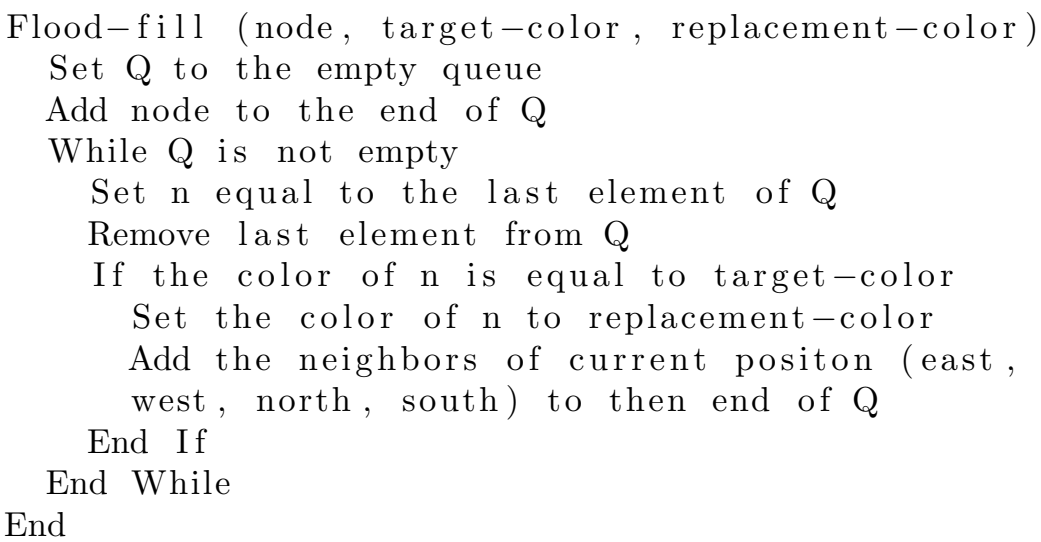

Removing Red Blood Cells. To remove red blood cells of muscle tissue a segmentation process is performed similar to the removal of connective tissue. Initially, a thresholding on the red channel is performed, obtaining a segmentation of the regions belonging to the red blood cells. About thresholding the result is applied to the morphological operation, erosion, in order not to be part of muscle tissue. After, regions under a threshold2 are removed to avoid segmented muscle or connective cells nuclei. Finally, the muscle tissue image is segmented to subtract the red blood cells from the image, the result of subtracting the second from the first is:

$$
I-(I \cap B)
$$

let $I$ be the muscle image segmentation. Let $\mathrm{B}$ be the red blood cells image segmentation. The regions under a threshold3 are removed to eliminate irrelevant regions resulting from the subtraction.

\section{Experiments and Analysis of Results}

In order to assess the proposed approach, loose connective and muscle tissues samples were processed with hematoxylin-eosin staining. The K-means algorithm for this particular case will use a $K=3$, each of these clusters will recognize: loose connective tissue, muscle tissue and light regions. The input for the K-means are: the red and the green color channels along with the tensor values obtained. Identification is evaluated by eye, evalution of experts. Automatic segmentation results can be observed in selected images in Fig. 2. 

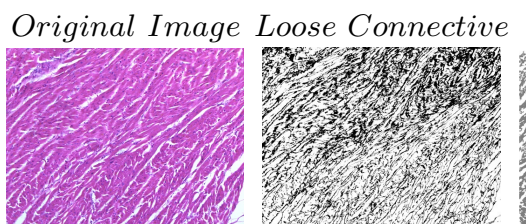

Muscle
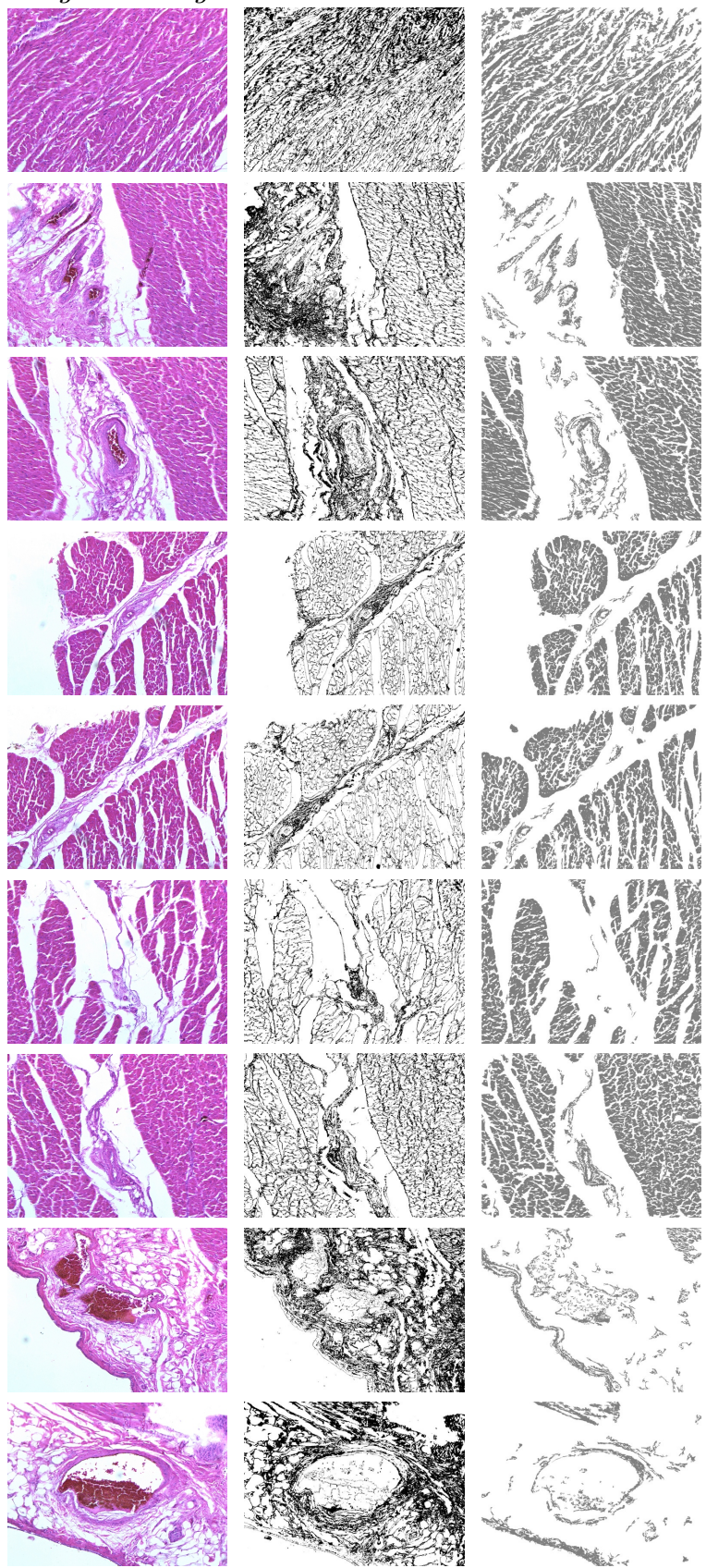

Fig. 2. Results obtained using automatic technique for identify loose connective and muscle tissues 


\section{Conclusions}

During the research process different approaches were evaluated, such as: thresholding, edge detection algorithms, combining information as input to the k-means algorithm and segmentation algorithms for identifying loose connective and muscle tissue. However, the presented approach provides the closest identification to the real identification - by eye - according to expert opinion.

The difficulty for identifing the loose connective tissue when is immersed in muscle tissue - even visually — is solved with the proposed method.

The experimental evaluation shows that the obtained segmentation is very close to the real one. Additional constrains are required in order to reduce false positive.

\section{References}

1. Rao, A.R., Schunck, B.G.: Computing oriented texture fields. In: Proceedings of the IEEE Computer Society Conference on Computer Vision and Pattern Recognition, CVPR 1989, pp. 61-68 (1989)

2. Weickert, J.: A Scheme for Coherence-Enhancing Diffusion Filtering with Optimized Rotation Invariance. Journal of Visual Communication and Image Representation 13, 103-118 (2002)

3. Kanungo, T., Mount, D.M., Netanyahu, N.S., Piatko, C.D., Silverman, R., Wu, A.Y.: An efficient k-means clustering algorithm: Analysis and implementation. IEEE Transactions on Pattern Analysis and Machine Intelligence 24, 881-892 (2002)

4. Nosal, E.-M.: Flood-fill algorithms used for passive acoustic detection and tracking. In: New Trends for Environmental Monitoring Using Passive Systems, pp. 1-5 (2008)

5. Lu, B., Miao, C., Wang, H.: Pixel level image fusion based on linear structure tensor. In: 2010 IEEE Youth Conference on Information Computing and Telecommunications (YC-ICT), pp. 303-306 (2010)

6. Vegue, J.B.: Atlas de Histología y Organografía Microscópica. Editorial Medica Panamericana, S.A. Madrid, España (2011)

7. Mazo, C., Trujillo, M., Salazar, L.: An Automatic Segmentation Approach of Epithelial Cells Nuclei. In: Alvarez, L., Mejail, M., Gomez, L., Jacobo, J. (eds.) CIARP 2012. LNCS, vol. 7441, pp. 567-574. Springer, Heidelberg (2012)

8. Haralick, R., Shapiro, L.: Computer and Robot Vision, vol. 1, ch. 5. Addison-Wesley Publishing Company (1992) 